\title{
Usefulness of VRML building models in a direction finding context ${ }^{*}$
}

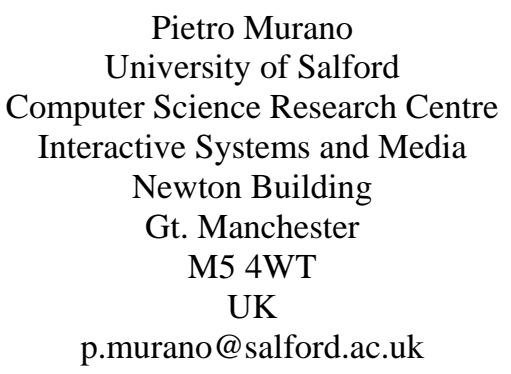

\author{
Dino Mackey \\ University of Salford \\ Computer Science Research Centre \\ Interactive Systems and Media \\ Newton Building \\ Gt. Manchester \\ M5 4WT \\ UK \\ dinomackey@hotmail.com
}

\begin{abstract}
This paper describes an experiment which aims to examine the effectiveness and efficiency of a Virtual Reality Modelling Language (VRML) building model compared with equivalent architectural plans, for direction finding purposes. The effectiveness and efficiency issues being primarily investigated were number of tasks completed overall and task completion times. The experiment involved a series of tasks where participants had to find a number of locations/objects in a building unknown to them at the outset of the experiment. Statistically significant results are presented for the benefit of the research community, law enforcement officers and fire fighters where it is clear that in this context, the VRML model led to better task completions than the equivalent architectural plans. Regarding the task completion times, no statistical significance was found. Given the current climate of security issues and terrorist threats, it is important that law enforcement officers have at their disposal the best information possible regarding the layout of a building, whilst keeping costs down. This also applies to fire fighters when rescuing victims. This experiment has shown that a VRML model leads to better task completions in direction finding.
\end{abstract}

\section{Keywords}

VRML, direction finding, user interfaces

\section{Introduction}

In the current modern world emergency services play a constant and important role in safeguarding security and dealing with general emergencies. Fire fighters have to deal with various situations involving fires in buildings of all kinds and navigating their way around these buildings (Leco, 2006). Law enforcement officers are constantly being challenged by new threats and new extreme terrorist acts, such as a building being under siege, e.g. (Beslan School Hostage Crisis, 2006). While such personnel are highly trained and make use of various technologies it would benefit them to always use the latest equipment or techniques available to aid their job.

One of the technologies available is the use of Virtual Reality (VR) to model buildings, such as public buildings or buildings of strategic importance. Having an accurate VR model of a building could help fire fighters 'learn' the layout of a building and positioning of key items

\footnotetext{
${ }^{*}$ A shorter version of this paper was presented at the HCII 2005 conference, Las Vegas, USA.
} 
in a building in a short period of time. Alternatively law enforcement personnel could 'learn' the layout of a building along with the positioning of key items for the unfortunate situation of a building being under siege. Sometimes terrorists have spent considerable time and effort in learning all they can about the layout of a building (U.S. Cavalry, 2006). This can possibly disadvantage law enforcement personnel who do not know the layout of a building.

\subsection{Related Applications Research and Work}

This section will consider some of the literature relevant to the context of the experiment reported in this paper. This deals with the areas of emergency services, direction finding and spatial understanding of 3D models.

A context where the use of VRML is showing itself to be useful is in the law enforcement area. Particularly concerning the use of 'crime maps' (Lodha et al, 1999) law enforcement departments have traditionally used a large map on a wall with coloured pins representing crime issues and thus showing the areas of a city most affected by certain crimes. These have been made electronic in nature (usually linked to a Geographical Information System (GIS)) thus allowing the crime distributions in a city to be viewed on a monitor. However these have usually been 2D in nature and therefore limiting in the help they can give (Lodha et al, 1999). The authors believe that VRML crime maps are more useful as they allow a better understanding of data. 3D VRML models allow users to explore the data by means of animations and various manipulation tools, e.g. tilting and rotating (Lodha et al, 1999).

An additional system discussed in (Abernathy and Shaw, 1998) merges topographical, satellite/aerial images and Global Position System (GPS) data into a VRML model. Their context was modelling a 'relay racecourse' (Abernathy and Shaw, 1998) in the state of California, USA. The idea was to give runners more information when they studied the course prior to running it. One of the ideas was to give runners, unfamiliar with the course, extra information by means of a VRML model, such as being able to see hills and other 3D aspects of the course. While the authors are satisfied with their results, they would like to see VRML improved to facilitate more real world applications development (Abernathy and Shaw, 1998).

One study where direction finding in an evacuation context is being conducted is in (Hisanaga et al, 2005). Their aim has been to transmit 3D evacuation images of some environment to a hand-held device, whilst trying to deal with the obvious issues of large file sizes on a hand-held device. They have had some success in reducing file sizes, however their work has not been fully evaluated, with only basic experimental participant questionnaire results being reported. The evaluation was informal without firm formal tasks. Whilst the work appears to be still in progress, the 'system' developed should be evaluated more rigorously in terms of effectiveness and user approval.

Considering the context of 'rescue' situations, various simple techniques are being used by emergency services. Some examples include police forces retaining and consulting floor plans of buildings such as schools and having 'resource officers' visiting certain schools on a daily basis so as to be familiar with the building and any changes that may occur to the internal layout (Scallan, 2004). Further the more sophisticated method of using VR in helping to train personnel in the kinds of situations mentioned above (and other contexts, e.g. $\mathrm{Li}$ et al (2000)) is being investigated/used by other organisations, e.g. the Sandia National 
Laboratories are using VR to 'familiarise law enforcement teams with scenarios' involving hostage rescues ('Sandia National Laboratories', 2005). Also in (Querrec et al, 2003) virtual environments are being used for training fire fighters. Further work in such training contexts can be seen in ('PoliceOne.com', 2005) and ('Virtra Systems', 2005). Most of this kind of work centres around scenarios etc.

In Constantine (2006) and Davis (2006) it is clearly indicated that fire services in certain situations tend to try and find out about the layout of a building as part of a building inspections routine (i.e. before any emergency has arisen).

In an informal interview with Constantine (the author of Constantine (2006)), it was indicated that fire crews are trained in a variety of ways. Some of these include using computer-based scenarios in classrooms. Other techniques include using purpose built buildings. Further, within some real environment, fire crews practice their direction finding by being blindfolded and asked to 'follow' a certain route by 'feel-and-touch'. Informally 'feel-and-touch' involves a crew member using a hand to 'feel' their way along a set of walls leading to various turnings in a building. Some of these aspects are not unique to the UK fire services, as is indicated in Trozzo (2006). This article reports on how fire services were able to use an abandoned building for real fire training. They went as far as filling the building with smoke and asking fire crews to complete certain scenario 'rescue' missions within the building.

The key aspect with these 'rescue' issues is the fact that fire services (and potentially law enforcement officers) are operating in a real 3D environment of which they may be unaware of the actual internal layout/configuration. These issues link with an experiment conducted by Schnabel and Kvan (2003), in an architectural setting. The experiment tested three conditions for 'cube building', where the aim was to study a cube that was constructed of a series of possible shapes of various colours. Then the participants had to reconstruct what they saw by using actual wooden blocks. Three small groups of participants were randomly allocated to either a 2D paper based plan of a cube configuration, a 3D model of the same cube configuration appearing on a PC screen (like a VRML model) or a 3D model of the same cube configuration in an immersive environment. The authors found that the $2 \mathrm{D}$ paper based plans group incurred the most accurate reconstructions with the wooden blocks. Also the participants in this condition studied the plans on a level-by-level basis but manifested a lack of understanding of the spatial volume. However the 3D models gave the participants more knowledge about the spatial volume and the overall 3D configuration of the shape. These findings suggested to us that a 3D model could help in direction finding for 'rescue' type situations, such as those faced by fire services. If the findings of the experiment by Schnabel and Kvan (2003) were applicable to other more realistic and larger contexts, then one would expect that direction finding tasks would be more effective and more efficient given a 3D model and compared with a 2D paper based architectural plan. This is because Schnabel and Kvan (2003) found that the participants in the 3D model groups had a better awareness of spatial volume and 3D configuration knowledge.

Another aspect suggesting that a 3D model may be superior (compared with 2D architectural plans) in a direction finding context was that Johnson (1998) indicated that generally fire fighters tend to have 'activist learning styles' (Johnson 1998). Johnson suggests that this pragmatically means that they learn better 'through direct experience', rather than perhaps reading books or sitting in a classroom listening to an instructor. 
This brief review has shown that some researchers are indeed trying to use 3D models to help individuals visualise certain contexts. However this review also shows that emergency services do still tend to use less modern methods, e.g. architectural plans and visiting buildings of importance for learning their layout. However not much work seems to have been done to directly address the issues dealt with in the experiment described below, which investigates improving direction finding results in an unknown environment. This suggests that this experiment is of value to other researchers working to develop 3D models for emergency services.

\section{The Direction Finding Experiment}

This experiment therefore makes a contribution in the area of direction finding training for 'rescue' missions linking specifically with the work by Schnabel and Kvan (2003). Hence the results of an experiment, conducted using a VRML (Virtual Reality Modelling Language) model of a university campus building. The main aim was to determine if the VRML model led to better task completions in direction finding within the building and shorter time completions for direction finding, compared with an equivalent architectural plan of the same building. The results are of interest to anyone involved with direction finding, particularly in the areas of fire fighting or law enforcement.

\subsection{Background Issues}

The experiment was carefully designed to ensure the conditions under examination were biased as little as possible.

The first step was to find a suitable building to model. The building used in the models was a campus building that was considered by the authors to be of a suitable size. This meant that it was not too large for both the purposes of modelling and experimental testing. Further, the experiment required a building unfamiliar to the prospective users. The building used fulfilled all these criteria.

The second step was to visually analyse the internal aspects of the building and to catalogue various features of the building, e.g. location of fire extinguishers (for the purposes of future task design).

The third step was to obtain accurate architectural diagrams of the building and then develop accurate external and internal models of the building, using the Virtual Reality Modelling Language (VRML).

\subsection{Users}

30 participants were recruited to take part in a between users experiment. 12 participants were female and 18 were male. Their average age was 32. Further, $96 \%$ of participants had a minimum of a high school standard education.

\subsection{Experimental Design}


A between users design was used for this experiment. The participants were divided into two groups of 15, where one group was assigned to the architectural plans condition and the remaining group was assigned to the VRML model condition.

\subsubsection{Task design}

The next aspect considered was the design of appropriate tasks that would elicit the information the experiment was trying to achieve. This was mainly to discover if a VRML model was more effective and efficient than an architectural diagram for direction finding tasks. Effectiveness for this context was defined as a participant obtaining higher scores in the assigned tasks (see next paragraph for details on the scoring mechanism and tasks used). Efficiency for this context was defined as leading to a quicker overall task completion time. These are in line with the International Organization for Standardization (1999) guidelines for usability. This particular standard states that effectiveness can concern issues of appropriate 'completeness' of goals - in this case completing the assigned tasks. For efficiency, the standard states that 'time' issues can be considered - in this case how long the tasks took to complete. Further it was also of interest to find out what strategies participants in each group would use to 'remember/learn' the layout of the building and to see if the strategies used would be similar to the strategies used by the participants in the study by Schnabel and Kvan (2003), given that the scenarios involved were significantly different.

A series of 16 tasks were designed which involved finding various locations and items within the chosen campus building. The particular locations/items were building evacuation refuge locations, fire escapes, fire extinguishers, fire alarm panels and fire hoses. Users finding specified locations/items would accrue a series of points. Some locations/items, due to where they were located in the building were considered to be easier to find and were therefore given fewer points than other locations/items considered to be more difficult to find. As stated a total of 16 tasks were designed. This allowed a sufficient number of 'easy' and 'more difficult' tasks to be used and also allowed for several areas of the building to be explored. The 16 tasks also made the experiment non-trivial as the participants had to use more 'mental effort'.

The points system used was weighted to take into account the fact that with certain locations/items finding one item would make it easier to find the next item. In these particular cases, the first find accrued more points than the second find. Also the particular locations/items used for the tasks were ones where finding them required some 'searching'/'recall', i.e. they could not be found by simply standing at the entrance of the building and visually scanning the immediate environment. An example concerning the building evacuation refuge locations is that the ones used in the experiment were on the first floor and second floor middle staircases. Whichever of the two was found first, 4 points were allocated and if the remaining refuge location was found, a further 2 points were allocated. This method allowed for different user direction finding strategies, not penalising one strategy against another. The points assigned to each location as explained above were to do with the overall difficulty of finding these locations.

Having decided on the tasks users should perform, the overall scores of the tasks would be used to see which of the modes being tested was better, i.e. each user's score would be summed to get one score and then all the scores would be analysed to see if one mode 
(VRML model or architectural plan) led overall to higher scores. Also users would be timed and the aim would be to see which mode would lead to faster completion times.

\subsection{Variables}

The independent variables were the modes of information presentation, i.e. architectural plans and VRML model.

The dependent variable consisted of the participants' performance in carrying out the designed tasks and the participants' subjective opinions regarding their learning strategy.

The dependent measures, for participant performance, were the timing of participants throughout the tasks undertaken and recording their success rate for each task by means of the scoring system devised (see previous sub-section - Task Design). The dependent measures, for participant learning strategy, were elicited by means of a post-experiment questionnaire.

\subsection{Apparatus and Materials}

The equipment used for the experiment was:

- Medium to high-end PC, capable of rendering a VRML model efficiently.

- A VRML plug-in.

- The 2 VRML models used in the pre-experiment screening and the actual VRML model of the campus building (see next section for details).

- The architectural plans for the pre-experiment screening and the actual campus building architectural plans (see next section for details).

- Pre-experiment screening test (see next section for details).

- Pre-experiment and post-experiment questionnaires.

\subsection{Experiment procedure}

Each participant was given a pre-experiment questionnaire which aimed to determine their personal experience and characteristics. Various questions were included in the questionnaire. The main questions dealt with issues of the participant's experience with computers, Virtual Reality and knowledge about building fire procedures. Participants were also asked if they had ever been in the campus building that was to be used in the experiment (i.e. only participants who had never been in the building were used). Clearly, if a prospective participant had prior knowledge about the building, it would invalidate any results for the experiment.

Having received completed questionnaires, the participants were then asked to take part in a pre-experiment screening. This aimed to determine/add to their current skills in either using VRML controls at the user interface or the reading of architectural plans in general. It also allowed the participants to see the kind of interaction they would have to be involved with when the experiment was started. The pre-experiment screening involved using a different VRML model and architectural plan to the ones used in the actual experiment. The VRML model used for this purpose was the main example used in (Hartman and Wernecke, 1996). 
The architectural plans used were John Adams' Courthouse MS USA ('The Historic Renovation of the John Adams Courthouse', 2004).

The participants using the architectural plans were given 20 minutes to study these. Then the plans were removed from the participants and subsequently were given 5 minutes to complete a multiple choice test, which covered various locations and characteristics of the Courthouse. If the participants obtained a score of $50 \%$ or more in the multiple choice test, they were considered to be suitable participants (15 participants passed the pre-experiment screening and were therefore used in the architectural plans group). This criterion was applied because in the real world individuals such as law enforcement officers etc, are expected to have certain skills to an appropriate level. Also this means that there should not have been extreme wide ranging levels of skill, which could have biased the final results.

The participants using the VRML model were selected as having a moderate amount of computer experience, as stated on the pre-experiment questionnaires they completed. They were given the VRML model and were asked to explore the model over a 20 minute period. During this time the participants were informally observed to ascertain their competence with the VRML navigational controls at the user interface. All participants in this group demonstrated competence in using the VRML controls.

As indicated above each participant was assigned to one of two groups. The first was the architectural plans group and the second was the VRML model group, dependent on the criteria described above.

The 15 participants selected for the architectural plans group were then given the diagrams for the campus building. Each participant was given 25 minutes to study the plans, which covered four floors in total. Relevant locations/items were clearly marked on the diagrams.

The 15 participants selected for the VRML model group were then asked to explore the VRML model of the campus building using the same VRML browser they had used during the pre-experiment screening process. Each participant was given 25 minutes to study the model. The model included the locations/items of relevance to the experiment. Further, locations were 'marked' in the model by means of white cubes containing location names.

The information presented in both modes (i.e. architectural plans and VRML model group) was identical. However the manner of the information presentation was different.

Once all participants (i.e. both groups) had completed the study of the campus building, each participant was briefed concerning what would happen in the next stage of the experiment. The following briefing points were made clear to each participant:

1) Participants would be physically accompanied/followed to the campus building (the technique of actually taking a participant to a real location was considered to be more realistic and has been successfully used and described in Murano (2003)) and be asked to find a series of locations/items based on their study of the campus building.

2) Participants would be asked to locate a subset of the locations/items they had seen on their preparatory study for the experiment.

3) Depending on the type of locations/items involved, the set of tasks would be divided into related sections. Thus the experiment would take place by completing a series of sections. 
4) Participants would be timed with a stopwatch.

5) Participants would have 2 minutes for each individual task. If this time was exceeded the particular task in question would be stopped and the next task would be started.

6) Participants would not be allowed to ask questions to aid their direction finding. Further, only 1 answer per task would be allowed.

7) If a location/item could not be found by the participant they could either attempt an answer or move to the next task.

8) Data would be recorded by the experimenter as the experiment tasks proceeded.

9) Participants would be able to ask clarifying questions regarding the briefing instructions described above.

At the end of the experiment all participants were asked to complete a post-experiment questionnaire. Certain questions were identical for both groups. However the VRML model group had a supplementary set of questions regarding the use of the VRML browser.

Both groups of participants were asked several subjective questions concerning how the participants felt they had 'learned' the locations for the tasks. They were also asked if in their opinion they had been given enough time to 'learn' the locations and actually find them during the experiment.

The VRML model group were also asked several subjective questions regarding the model itself, e.g. accuracy of the model in relation to the real building and also questions regarding the ease of use of the model. Further, they were asked their opinions regarding the issue of 'learning' locations by the specific use of the VRML model.

\subsubsection{Pilot study}

Before the actual experiment took place a small pilot study was undertaken to test the experimental design for soundness. One of the reasons for having included a pre-screening stage to the experiment was that the pilot study revealed that in the VRML model condition a usage of time was incurred in becoming familiar with the particular VRML browser used in the experiment. Thus the pre-screening process gave the participants the opportunity of familiarising themselves with the VRML controls thus lessening the possibility of bias during the actual experiment.

Also, being able to run through the experiment, allowed the maximum times to be established for each task. These were set to 2 minutes and based on what was observed during the pilot study, this was considered to be a reasonable amount of time for each task. The time of 2 minutes was actually more than was required for the completion of a task. This would allow for participants who may have been naturally slightly slower in nature.

\subsection{Results}

Some interesting and useful results were obtained. Firstly, regarding the overall times taken to complete the tasks for the VRML model condition $(\mathrm{M}=723.87$; $\mathrm{SD}=152.29)$ and the architectural plans group $(\mathrm{M}=693.73$; $\mathrm{SD}=116.39)$, a t-test showed no statistically significant results beyond the 0.05 level $-/ \mathrm{t} /(28)=-0.61, / \mathrm{p} /=0.55$. Table 1 below shows the figures in tabular format. 
However the rate of success in the task completions was higher under the VRML model condition. Participants achieved higher scores under the VRML model condition $(\mathrm{M}=$ 44.93; $\mathrm{SD}=6.66$ ), i.e. the tasks were more successfully completed under this condition, compared to the architectural plans condition $(\mathrm{M}=39.33$; $\mathrm{SD}=6.91)$. A t-test showed statistically significant results beyond the 0.05 level $-/ \mathrm{t} /(28)=-2.26, / \mathrm{p} /=0.03$. Table 2 below shows the figures in tabular format.

Secondly, subjective opinions were elicited from the participants by means of a postexperiment questionnaire. The questionnaire was designed as a series of questions accompanied by a set of answers on a Likert scale, where 1 was a completely negative response and 5 was a completely positive response.

The most interesting and principal aspects concerned the participants' impressions of how they 'learned'. The users of the VRML model reported not actually memorising the items/locations $(M=2.13 ; S D=1.13)$. They grouped the items/locations together $(M=4.00$; $\mathrm{SD}=1.20)$ and did not pay any attention to compass directions $(\mathrm{M}=2.13$; $\mathrm{SD}=1.53)$. The users of the plans instead reported learning the positions of items/locations one floor at a time $(\mathrm{M}=4.90 ; \mathrm{SD}=0.40)$. They did not group items/locations together $(\mathrm{M}=2.00 ; \mathrm{SD}=$ $0.70)$ and they actually used the compass directions on the plans $(\mathrm{M}=4.60$; $\mathrm{SD}=0.51)$. The figures are shown in tabular format in Tables 3 and 4 below.

Other aspects regarding the participants' subjective responses indicate that both experimental groups showed agreement concerning the fact that they built a 'mental picture' of the building from their initial study (VRML model group $\mathrm{M}=4.53$; $\mathrm{SD}=0.49$, Architectural plans group $\mathrm{M}=4.60$; $\mathrm{SD}=0.51$ ) and that they felt they had a 'clear picture' of the building layout (VRML model group $\mathrm{M}=4.87 ; \mathrm{SD}=0.35$, Architectural plans group $\mathrm{M}=4.60 ; \mathrm{SD}=$ $0.51)$.

Also, most users in both experimental groups felt that they had been given enough time to study the architectural plans/VRML model (VRML model group $\mathrm{M}=4.20 ; \quad \mathrm{SD}=0.78$, Architectural plans group $\mathrm{M}=3.87$; $\mathrm{SD}=1.13$ ) and carry out each task (VRML model group $\mathrm{M}=4.00 ; \mathrm{SD}=1.13$, Architectural plans group $\mathrm{M}=4.27 ; \mathrm{SD}=0.46$ ). They also tended to agree that the representation of each location/item was easy to understand (VRML model group $\mathrm{M}=4.60 ; \mathrm{SD}=0.51$, Architectural plans group $\mathrm{M}=4.40 ; \mathrm{SD}=0.51$ ).

Finally, the VRML model group indicated clearly that the accuracy of the VRML model in relation to the real building was to a very good standard and that the VRML model was easy to use.

\section{Discussion}

As can be seen from the previous section this experiment has resulted in various achievements. The first achievement shows that there is no statistically significant evidence to suggest that the VRML model group was faster than the architectural plans group with respect to task completions. Therefore the efficiency aspect is not significantly affected.

The aspect concerning speed of task completions and the lack of statistical significance simply shows that the two groups were well balanced in terms of ability to travel around an 
environment on foot. This had not been a specific requirement for the experiment. However no user taking part in the experiment was physically impaired in any way.

The second achievement shows that the task completion rate was higher under the VRML model group with statistically significant results suggesting that a VRML model, in this context, leads to better task completions. Therefore in this context the effectiveness is significantly affected in favour of the VRML model. This is because the tasks involving direction finding made use of the spatial volume and the overall 3D configuration knowledge of the building as discovered by Schnabel and Kvan (2003) in their study of rebuilding a cube. A further reason for this observed result could be that the participants had a greater sense of 'presence' with the VRML model. According to Huang and Alessi (1999), 'presence' has various definitions, including the feeling of being in a virtual environment. They also state that human emotions affect behaviour and are linked with feelings of 'presence' (Huang and Alessi, 1999). Although their discussion is more related to immersive environments, the emotional aspect could be an explanation for the more successful rate in task completions. It may be the case that the VRML model fostered a greater emotional response and a better feeling of 'presence', which in turn led to better task completions as the VRML model was more easily 'mapped' to the real environment. This aspect however would require further examination by means of further similar studies. Nevertheless, this is a very important finding as a higher rate of task completions in a real world could potentially save lives, particularly in the context of fire fighting and/or law enforcement. Some examples of this could be fire fighters being able to find an exit of a building or a specific room full of people in a building. Also law enforcement officers may be able to gain the element of surprise on a terrorist group in a building by using certain routes through the building.

The third achievement concerns the way the participants reported their manner of 'remembering/learning'. Tables 3 and 4 show interesting participant self-reported learning mechanisms. These all have either quite low or very low accompanying standard deviations. These show a high consistency in the participant responses. This suggests that participants in each of the two groups were using similar 'learning' strategies, dependent on the mode of information presentation. Specifically, the architectural plans group reported learning the environment by memorising and on a floor-by-floor basis. This is consistent with the findings of Schnabel and Kvan (2003), where their participants in the 2D floor plans group learned about the cube on a level-by-level basis and by memorising. The VRML model group in the direction finding experiment reported learning the environment 'automatically' and by grouping locations/objects together. This is also consistent with the findings of Schnabel and Kvan (2003), where the two groups using 3D models learned and reconstructed the cube as 'one element'. Further, they showed a better awareness of neighbouring elements making up a cube, i.e. groups of elements. This suggests that the 'remembering/learning' strategies of users tend to be similar despite the dramatic change in context. This aspect is also of interest because if it is known how most users learn a location based on the type of information given to them (e.g. via architectural plans or VRML models), the instructors in training situations can use this to help fire crews or law enforcement officers leverage these aspects to their advantage. They would achieve this by aligning their training sessions appropriately to such modes of learning.

The uses for this experimental finding are that it could help fire fighters (or other emergency services) improve their direction finding skills. Also if models were widely available for 
public buildings, it could help law enforcement personnel to deal with a worst case scenario of a terrorist siege. The suggestion being made is not to abandon current training/practices. However, these should be augmented by the use of VRML models as they give the user a valuable insight not available with architectural plans and the experiment indicates that the internals of a building are learned in a more useful way than with architectural plans on their own. VRML models of key buildings should be available for fire fighters and law enforcement officers.

One drawback of this suggestion is that VRML models would have to be constantly kept up to date as sometimes the internal configuration of buildings can change due to refurbishment etc. Also using such models may necessitate the development of models at the time a building is designed. These could then be lodged with the usual architectural plans. Furthermore there would be a cost factor involved in developing such models both in terms of money and time. Lastly it may not be practical to have certain buildings modelled in this way due to perhaps the confidential nature of the work being carried out in the building (e.g. a cabinet minister's suite of offices).

A further aspect this experiment has shown is that powerful useful visual information can be conveyed to users with basic equipment and software (i.e. not specialist hardware/software). Once the cost of building a model is considered, it would be simple to have a model on a laptop PC and carry this to any appropriate location. Linked to the issue of costs is the fact that VRML models are essentially 3D models. Such 3D models can be built in different ways. One way is from first principles, which requires more specialist knowledge. However, 3D building tools, e.g. Autodesk 3ds Max (2006), are also available thus making the building of a 3D world more accessible to non-computer scientists. Another aspect is that public services, such as fire brigades, tend to be on a limited budget and this proposed solution would be relatively cost effective.

Overall the mainly positive results of this experiment continue to support the trend of positive results already published in this area, such as the ones discussed above, by Lodha et al (1999) and Abernathy and Shaw (1998). These are showing generally positive findings in favour of using 3D models in various contexts.

\section{Further work}

The experiment generated some interesting results. However the experiment also raised issues requiring further investigation. The first issue concerns the campus building modelled in VRML. As stated above, the campus building was not a huge intricate building, even though it was big enough to conduct the experiment. It would therefore be interesting to find out if the same results would be obtained with a larger more intricate building, such as a public government building. This would in turn require scenarios to be designed that would last longer and fully exercise the direction finding skills of rescue services.

The second issue would be to involve real fire fighters and law enforcement officers in further experiments, perhaps including various emergency scenarios and different environments. If such individuals would be available, ideally they should be more closely screened regarding their backgrounds and general learning styles. These aspects could be determined by using 'psychological or cognitive screening'. These would help to strengthen 
future experimental results. Unfortunately fire fighters and law enforcement officers were not available for this work.

The third issue concerns the fact that the study and data collected would have been better if more participants could have been recruited. However, the authors take the stance that the number of participants, 15 in each group, is considered to be statistically moderate in size (Weiss, 1999) and therefore pragmatically acceptable for the purposes of this experiment.

The fourth issue involves the scores obtained concerning the participants' perceived selflearning mechanisms. As can be seen in the results section above, users in each of the two groups consistently showed themselves to be adopting similar learning strategies depending on which group they had been assigned to. It would be useful to find out if this effect was due to participants subconsciously adjusting their manner of learning depending on the mode of presentation of the information. This issue, as mentioned above, could also involve screening users concerning their own particular learning strategies. This in turn would require linking with existing learning theories and/or other issues of human cognition. If it could be determined that certain styles of learning affect the recall of an 'environment', fire fighters and law enforcement officers could be screened in advance so that in an emergency situation they could be given a particular type of visual information based on their learning style. Advance screening could take place as part of the recruitment process and/or part of any 'psychometric' type testing that some employers already deploy. Some of these issues could be resolved by conducting a further experiment(s) dealing directly with the cognitive and psychological issues mentioned above.

\section{Conclusions}

This paper has presented the results of an experiment conducted in the area of direction finding. The experiment tested a VRML model condition and an equivalent architectural plan condition. The main findings showed that the VRML model led to better task completions in a direction finding context. However, there was no difference between the two conditions in the actual time taken to complete the tasks. Participants in the experiment, depending on which condition they tested, reported similar within condition 'learning' strategies. The findings of this experiment are of use and interest particularly to fire fighters or law enforcement officers. This is because having a VRML model of a building could aid their task of finding a location in an emergency. Despite these findings, the work presented in this paper needs to be taken further by investigating in more detail the human learning strategies when using a VRML model and to use real fire fighters or law enforcement officers in further experiments.

\section{Acknowledgements}

The authors would like to thank Computing, Science and Engineering at the University of Salford and particularly Prof. Tim Ritchings and Prof. Sunil Vadera for their support in various aspects of this research. Also Dr. Farid Meziane for his advice in preparing the journal version of this paper.

\section{References}


Abernathy, M. \& Shaw, S. Integrating Geographic Information in VRML Models. VRML 98 Symposium, Monterey, California, 16-19 Feb, (1998) ACM.

Autodesk, http://usa.autodesk.com/adsk/servlet/item-w?siteID=123112\&id=2774123, (2006). Accessed 2006.

Beslan School Hostage Crisis, http://en.wikipedia.org/wiki/Beslan_school_hostage_crisis, (2006) Accessed 2006.

Constantine, N., 24 Hours in the Life of a Firefighter, http://www.firerescue.homestead.com/firefightersday.html. (2006). Accessed 2006.

Davis, K. Burning Desire to Help: CFFA Graduates Confident, Professional Firefighters, The Maple Leaf,

http://www.forces.gc.ca/site/Community/mapleleaf/index_e.asp?newsID=2855\&id=5701\&c at=5\&volID=1\&issID=48\&newsType=1 (2006). Accessed 2006.

Hartman, J. \& Wernecke, J., The VRML 2.0 Handbook Building Moving Worlds on the Web. Addison Wesley, 1996.

Hisanaga, S. Suganuma, Y, Maehara, H. Wakimoto, K. Tanaka, S. Evacuation Route Guidance via Mobile Phones based on Three-dimensional Geographical Information Delivery. $11^{\text {th }}$ International Conference on Human Computer Interaction, Las Vegas, USA, 22-27 July (2005).

The Historic Renovation of the John Adams Courthouse, Architectural Plans, http://renovation.socialaw.com/archplans.htm, (2004), Accessed 2006.

Huang, M. P. and Alessi, N. E. Presence as an Emotional Experience, Medicine Meets Virtual Reality: The Convergence of Physical and Informational Technologies Options for a New Era in Healthcare: The Convergence of Physical and Informational Technologies Options for a New Era in Healthcare, Edited by Westwood, J.D., Hoffman, H.M. and Stredney, D., IOS Press, (1999) p. 148-153.

International Organization for Standardization, Ergonomic Requirements for Office Work With Visual Display Terminals Part 11: Guidance on Usability, http://www.iso.org (1999). Accesses 2006.

Johnson, C. Evaluating the Contribution of Desktop VR to Computer Aided Learning, Workshop on Usability Evaluation for Virtual Environments, (1998) p. 69-79.

Leco, M. World Trade Center, http://www.usatourist.com/english/places/newyork/worldtradecenter.html, (2006) Accessed 2006.

Li, Y., Brodlie, K., Phillips, N. Web-Based VR Training Simulator for Percutaneous Rhizotomy, Medicine Meets Virtual Reality, Edited by Westwood, J.D., Hoffman, H.M., Mogel, G.T., Robb, R.A. and Stredney, D., IOS Press, (2000) p. 175-181. 
Lodha, S.K. Suresh, K. and Verma, A. Animations of Crime Maps Using Virtual Reality Modelling Language. Western Criminology Review 1 (2) [online]

http://wcr.sonoma.edu/v1n2/lodha.html. (1999) Accessed 2006

Murano, P., Anthropomorphic Vs Non-Anthropomorphic Software Interface Feedback for Online Factual Delivery, $7^{\text {th }}$ International Conference on Information Visualisation (IV 2003) An International Conference on Computer Visualisation and Graphics Applications, London, England, 16-18 July, (2003) @ IEEE

PoliceOne.com, Advanced Interactive Systems, Inc. Develops National Training Program.http://www.policeone.com/police-products/training/press-releases/88222/ (2005) Accessed 2006.

Querrec, R., Buche, C., Maffre, E., Chevallier, P., SecuReVi: Virtual Environments For Fire Fighting Training, VRIC 2003 (Colloque Laval Virtual 2003 Cinquième Conférence Internationale Sur La Réalité Virtuelle), Laval, France, 14-16 May (2003) p. 169-175.

Sandia National Laboratories, Hostage Rescues Honed in Virtual Reality Simulation, http://www.sandia.gov/media/vr.htm - (2005) Accessed 2006

Scallan, M.M., Terrorist Threat Poses Challenge for Mississippi Schools, Biloxi Sun Herald via Associated Press http://www.officer.com/article/article.jsp?id=16882\&siteSection=8. 10.9.04 - (2004) Accessed 2006

Schnabel, M.A. \& Kvan, T. Spatial Understanding in Immersive Virtual Environments, International Journal of Architectural Computing, 1(4) (2003).

Trozzo, S. Emergencies Take Practice, Pittsburgh Post-Gazette, http://www.postgazette.com/pg/06169/698646-54.stm (2006) Accessed 2006.

U.S. Cavalry, The Terrorist Attack Cycle: Selecting the Target, http://www.uscav.com/uscavonpoint/Feature.aspx?id=141, (2006) Accessed 2006.

Virtra Systems, http://www.virtrasystems.com. (2005) Accessed 2006.

Weiss, N. A. Introductory Statistics, Addison Wesley, (1999).

Table 1: T-test - Task Times - VRML Model and Architectural Plans Conditions

\begin{tabular}{ll}
\hline DF & 28 \\
Mean (VRML Model group) & $723.87 ;$ SD 152.29 \\
Mean (Architectural Plans group) & $693.73 ;$ SD 116.39 \\
/t/ & -0.61 \\
/p/ & 0.55 \\
Significance Level & 0.05
\end{tabular}


Table 2: T-test - Task Completion Success - VRML Model and Architectural Plans Conditions

\begin{tabular}{ll}
\hline DF & 28 \\
Mean (VRML Model group) & $44.93 ;$ SD 6.66 \\
Mean (Architectural Plans group) & $39.33 ;$ SD 6.91 \\
/t/ & -2.26 \\
/p/ & 0.03 \\
Significance Level & 0.05
\end{tabular}

Table 3: Participants’ Subjective ‘learning’ impressions (VRML Model Group)

\begin{tabular}{lcc}
\hline & Mean & SD \\
& & \\
Mental Picture Built of Building From Initial Study & 4.53 & 0.49 \\
Clear Picture of Building Layout From Initial Study & 4.87 & 0.35 \\
& & \\
Locations Memorised & 2.13 & 1.13 \\
Locations 'Automatically' Remembered & 4.00 & 1.00 \\
Locations/Objects Learned on Floor-by-Floor Basis & 2.60 & 1.30 \\
Locations/Objects Learned by Grouping Each Type Together & 4.00 & 1.20 \\
Locations/Objects Learned by Use of Compass Directions & 2.13 & 1.53 \\
& & \\
Enough Time Given to Study the Model & 4.20 & 0.78 \\
Enough Time Given for Each Task & 4.00 & 1.13 \\
Representation of Locations/Items Easy to Understand & 4.60 & 0.51 \\
& & \\
\hline
\end{tabular}

Table 4: Participants’ Subjective ‘learning’ impressions (Architectural Plans Group)

\begin{tabular}{lcc}
\hline & Mean & SD \\
Mental Picture Built of Building From Initial Study & 4.60 & 0.51 \\
Clear Picture of Building Layout From Initial Study & 4.60 & 0.51 \\
& & \\
Locations Memorised & 4.70 & 0.49 \\
Locations 'Automatically' Remembered & 1.90 & 0.64 \\
Locations/Objects Learned on Floor-by-Floor Basis & 4.90 & 0.40 \\
Locations/Objects Learned by Grouping Each Type Together & 2.00 & 0.70 \\
Locations/Objects Learned by Use of Compass Directions & 4.60 & 0.51 \\
& & \\
Enough Time Given to Study the Plans & 3.87 & 1.13 \\
Enough Time Given for Each Task & 4.27 & 0.46 \\
Representation of Locations/Items Easy to Understand & 4.40 & 0.51 \\
\hline
\end{tabular}

